\title{
Artigo Original / Original Paper \\ Influência de povoamento de Pinus taeda sobre o banco de sementes na região de Floresta Ombrófila Mista
}

\author{
Influence of Pinus taeda plantation on the seed bank in an Araucaria Rainforest region
}

\author{
Rubia Tatiana Secco ${ }^{1,3,4}$, Christopher Thomas Blum ${ }^{2}$ \& Santiago José Elías Velazco ${ }^{1}$
}

\begin{abstract}
Resumo
Este estudo teve o objetivo de avaliar o efeito de um povoamento de Pinus taeda sobre a composição florística e estrutura do banco de sementes de um remanescente de Floresta Ombrófila Mista (FOM). Para tanto foram coletadas 160 amostras do banco de sementes, 80 de um remanescente de FOM e 80 em um povoamento de P. taeda. As amostras foram colocadas para germinar em duas casas de vegetação distintas, sob 35\% e 75\% de níveis de sombreamento. Foram contabilizados ao todo 11.669 indivíduos lenhosos e herbáceos, distribuídos em 31 famílias botânicas e 84 espécies. As famílias Asteraceae e Poaceae foram as mais representativas em riqueza de espécies herbáceas. Dentre as lenhosas, Solanaceae e Asteraceae foram as mais representativas em ambas as áreas. A espécie $P$. taeda foi registrada apenas no banco de sementes do próprio povoamento. $\mathrm{O}$ banco de sementes do povoamento de pinus caracterizou-se principalmente pela baixa riqueza de espécies lenhosas, o que demonstra sua influência sobre o estoque de sementes. Por outro lado, o remanescente de FOM avaliado parece não sofrer influência da proximidade do povoamento de pinus no seu banco de sementes.

Palavras-chave: floresta com araucária, povoamento florestal, regeneração natural.
\end{abstract}

\begin{abstract}
This study aimed to evaluate the effect of a Pinus taeda plantation on the floristic and species composition of the seed bank in remnant of the Araucaria Rainforest (FOM). For this purpose, a total of 160 samples were collected from the seed bank, 80 of a FOM remnant and 80 from a $P$. taeda plantation. The samples were placed to germinate in two different greenhouses, under $35 \%$ and $75 \%$ shading level. A total of 11,669 woody and herbaceous individuals were counted, distributed in 31 botanical families and 84 species. The families Asteraceae and Poaceae were the most representative in richness of herbaceous species. With regard to woody species, Solanaceae and Asteraceae were the most representative in both areas. The species $P$. taeda was recorded only in the seed bank of the plantation. The seed bank of the pinus plantation was characterized mainly by the low richness of woody species, which shows its influence on the seed stock. On the other hand, the FOM remnant seems not to be influenced by the proximity of the pinus plantation to it is seed bank.

Key words: araucaria rainforest, planted forest, natural regeneration.
\end{abstract}

\section{Introdução}

A Floresta Ombrófila Mista (FOM), unidade fitogeográfica característica dos planaltos do Sul do Brasil, é marcada pela dominância da espécie Araucaria angustifolia (Bertol.) Kuntze, que divide o dossel com exemplares de Lauraceae e Meliaceae, apresentando uma estimativa de diversidade florística arbórea superior a 350 espécies em toda a região fitoecológica (Roderjan et al. 2002). No entanto, devido à forte exploração madeireira do século XX, principalmente da araucária e de outras madeiras de alto valor econômico, a FOM que já ocupou quase metade do território do estado do Paraná, hoje se encontra próxima à extinção

\footnotetext{
${ }^{1}$ Universidade Federal do Paraná, Prog. Pós-graduação em Engenharia Florestal, Campus III, Av. Lothário Meissner 631, Jardim Botânico, 80210-170, Curitiba, PR, Brasil.

${ }^{2}$ Universidade Federal do Paraná, Depto. Ciências Florestais, Campus III, Av. Lothário Meissner 631, Jardim Botânico, 80210-170, Curitiba, PR, Brasil.

${ }^{3}$ ORCID: <https://orcid.org/0000-0003-3873-6235>

${ }^{4}$ Autor para correspondência: rubia.secco@gmail.com
} 
(Kersten et al. 2015). Desta forma, a preservação dos remanescentes da FOM é imprescindível, sendo a compreensão dos processos de regeneração natural de grande interesse neste contexto (Narvaes et al. 2005), especificamente a condução dos bancos de sementes de solos florestais (Seubert et al. 2016).

O banco de sementes é considerado como um dos principais mecanismos da regeneração natural das espécies tropicais (Garwood 1989), este consiste em um depósito de sementes viáveis provenientes das plantas locais e daquelas vindas por diferentes meios de dispersão (Garwood 1989; Dalling et al. 1998). A existência deste depósito de sementes garante que a floresta será renovada a cada perturbação, podendo em alguns casos promover o aumento da biodiversidade vegetal (Oliveira Júnior \& Constantin 2001). O período de permanência destas sementes no solo depende de uma série de fatores que variam de fisiológicos (dormência) a ambientais ou ecológicos - luminosidade, temperatura, umidade, predação, entre outros (Garwood 1989; Lopes et al. 2006).

Alguns estudos têm mostrado que, em monoculturas de espécies arbóreas exóticas, o banco de sementes no solo é dominado por espécies herbáceas e outras espécies pioneiras características de formações iniciais de sucessão ecológica, sendo que quanto maior a intervenção nos povoamentos maior a proporção de indivíduos herbáceos (Gonçalves et al. 2008; Nóbrega et al. 2009). Entre as espécies arbóreas amostradas nestes bancos de sementes, exemplares das espécies do próprio plantio florestal, sobretudo dos gêneros Pinus e Eucalyptus, encontram-se presentes na maioria dos casos avaliados (Ne'eman \& Izhaki 1999; Gonçalves et al. 2008; Schorn et al. 2013).

A espécie Pinus taeda L., plantada em larga escala no Sul do Brasil, é caracterizada como exótica invasora, podendo ser encontrada colonizando diferentes ambientes como campos naturais, cerrados, florestas secundárias e ambientes perturbados (Espíndola et al. 2015; GISP 2005). A invasão acontece uma vez que esta espécie apresenta atributos que a permitem reproduzir-se a ponto de ocupar o espaço de espécies nativas, produzindo assim alterações nos processos ecológicos naturais e tornando-se com o passar do tempo dominante nestes ecossistemas (Ziller \& Galvão 2002).

Diante deste cenário, este trabalho objetivou caracterizar o banco de sementes de duas áreas próximas, uma em remanescente secundário de
FOM no estágio intermediário de sucessão, e outra em povoamento de Pinus taeda, a fim de verificar os efeitos de um plantio florestal de P. taeda na composição florística, riqueza e estrutura do banco de sementes autóctone em região de Floresta Ombrófila Mista.

\section{Material e Métodos}

Área de estudo

As amostras de solo, para análise dos bancos de sementes, foram coletadas no município de Campo do Tenente, no estado do Paraná, em um remanescente secundário de Floresta Ombrófila Mista (2600'14.65'S, 4945'18.74”O - 855 m a.n.m.) em estágio médio da sucessão (Brasil 1994) e sem indícios de invasão por pinus, e em um povoamento comercial de Pinus taeda, com 15 anos de idade e em franca produção de sementes (2600'10.55"S, 4944'39.95"O - 825 m a.n.m.), distando aproximadamente $1 \mathrm{~km}$ entre si.

Aárea de estudo situa-se no segundo planalto, na porção sudeste do estado. A geologia local é caracterizada pelo Grupo Itararé, formação Campo do Tenente, representada pelos arenitos da Lapa (MINEROPAR 2001). Os solos predominantes na região são Neossolos, Latossolos e Cambissolos (EMBRAPA 2012). A formação florestal original é a Floresta Ombrófila Mista, com presença de relictos de campos naturais (ITCG 2009).

A imagem aérea das áreas de estudo, datada de 1980 (ITCG 2015), mostra que ambas eram cobertas por vegetação nativa naquela época. A área com a espécie exótica encontra-se em seu segundo plantio de pinus, sendo o primeiro removido quando o povoamento atingiu 15 anos de idade.

O clima da região, segundo a classificação de Koppen, é Cfb - temperado úmido mesotérmico (IAPAR 2016a). A média da temperatura anual da região é $18,2{ }^{\circ} \mathrm{C}$, sendo $23,2{ }^{\circ} \mathrm{C}$ e $13,2{ }^{\circ} \mathrm{C}$ as médias das temperaturas máximas e mínimas, respectivamente, a precipitação média anual é de $1.645 \mathrm{~mm}$, sendo janeiro o mês mais chuvoso e agosto o de menor precipitação (IAPAR 2016b).

\section{Amostragem do banco de sementes}

Para a coleta de amostras do banco de sementes em cada uma das tipologias vegetais foram estabelecidos quatro transectos paralelos de $100 \mathrm{~m}$ de comprimento e equidistantes $20 \mathrm{~m}$ entre si, abrangendo áreas homogêneas e representativas de cada tipologia, e obedecendo a uma distância de $30 \mathrm{~m}$ da borda do fragmento, tendo em vista eliminar interferência do efeito de borda. Em cada 
transecto foram coletadas amostras aos pares a cada $10 \mathrm{~m}$. Quando no ponto predeterminado de coleta havia algum impedimento (rochas, raízes expostas, entre outros), esta foi realizada a $2 \mathrm{~m}$ do ponto pré-estabelecido, desde que se mantivesse a homogeneidade ambiental, seguindo a seguinte sequência de hierarquização: $1^{\circ}$ ) $2 \mathrm{~m}$ a frente do ponto inicial da coleta; $2^{\circ}$ ) $2 \mathrm{~m}$ para trás; $\left.3^{\circ}\right) 2 \mathrm{~m}$ à direita em ângulo perpendicular ao transecto; ou, $\left.4^{\circ}\right) 2 \mathrm{~m}$ à esquerda em ângulo perpendicular ao transecto. Ao todo foram coletadas 20 amostras por transecto, totalizando 80 amostras por tipologia vegetal.

A coleta foi realizada com o auxílio de um gabarito de $25 \times 25 \times 6 \mathrm{~cm}$, o qual foi inserido no solo para delimitar a área amostral. A serapilheira não decomposta foi retirada e descartada previamente ao procedimento de coleta do substrato.

As amostras de substrato foram colocadas em bandejas plásticas de $32 \times 25 \times 13 \mathrm{~cm}$, contendo uma camada de $3 \mathrm{~cm}$ de pedra brita, para auxiliar na drenagem, e outra camada de $5 \mathrm{~cm}$ de substrato e vermiculita expandida, na proporção 4:1, misturados previamente. As bandejas foram alocadas em duas casas de vegetação, situadas no campus III da UFPR, Curitiba-PR, em região com as mesmas condições climáticas da área de estudo. Foram colocadas 40 amostras de solo da área do povoamento de pinus e 40 amostras de solo do remanescente florestal em casa de vegetação contendo cobertura de plástico transparente e sombrite com $75 \%$ de sombreamento, e o mesmo número de amostras em outra casa de vegetação apenas sob cobertura de plástico transparente, resultando em 35\% de sombreamento. Foram utilizados dois níveis de luminosidade a fim de proporcionar condições de germinação para o maior número de espécies possível. Os níveis de sombreamento foram medidos com o auxílio de Luximetro, sendo realizadas mensurações no interior das casas de vegetação e ao ar livre para conclusão dos resultados.

As bandejas foram regadas por aspersores quatro vezes ao dia, durante períodos de 10 a $15 \mathrm{~min}$ cada vez, o que foi ajustado conforme a necessidade promovida pelas condições meteorológicas garantindo condições adequadas para germinação das sementes, semelhantes às condições naturais. Para avaliar eventuais contaminações com sementes do entorno do experimento foram deixadas duas bandejas com substrato (caixas-controle) em cada casa de vegetação, monitoradas periodicamente.
A avaliação e quantificação das sementes germinadas foram realizadas semanalmente por doze meses. Em cada avaliação de monitoramento foram incluídas na contagem plântulas de espécies lenhosas (arbustivas, arbóreas e lianas) que apresentaram altura superior a $5 \mathrm{~cm}$. Para as espécies herbáceas, estas foram contabilizadas e coletadas com $10 \mathrm{~cm}$ de altura, após a identificação do primeiro exemplar de cada espécie. Após a contabilização do exemplar, este foi removido do experimento.

A identificação das espécies foi realizada com o auxílio de bibliografia especializada e comparação com material de referência dos Herbários EFC (Escola de Florestas Curitiba) e MBM (Museu Botânico Municipal de Curitiba). Plântulas que não puderam ser identificadas foram cultivadas em vasos até que se desenvolvessem a ponto de apresentar características morfológicas que permitissem sua identificação. Todo material botânico coletado fértil foi tombado no Herbário EFC.

As espécies foram classificadas em herbáceas e lenhosas, segundo as especificações determinadas por Martins \& Batalha (2013), sendo consideradas espécies herbáceas aquelas que geralmente possuem caule fino, flexível e coberto por uma epiderme lisa e fina, em geral na cor verde. Conforme os mesmos autores, foram consideradas espécies lenhosas, aquelas que possuem caule grosso, de madeira, rígido e coberto por uma casca, cujo aspecto externo pode ser liso, rugoso, estriado, com ou sem escamas, costas ou placas. Como várias destas características não se apresentam na fase juvenil, após a identificação da espécie, foi consultada a literatura com base nas características dos indivíduos adultos.

\section{Análise dos dados}

Para avaliação dos tratamentos (bancos de sementes da FOM e do povoamento de pinus) foram consideradas as parcelas sem e com sombreamento como um conjunto único, sendo avaliados os parâmetros: riqueza de espécies, frequência, densidade, e percentual de importância adaptado (PI), ou seja, baseado na média dos valores relativos de frequência e densidade. Para a análise de similaridade florística do banco de sementes foi utilizado o índice de similaridade de Jaccard.

A fim de avaliar diferenças na diversidade entre as duas áreas foram utilizados perfis de diversidade. Nestes a diversidade é expressa 
em um gradiente que varia segundo o aumento da importância da equitatividade no cálculo da diversidade, a qual depende do "q" (Chao \& Jost 2015). Os perfis de diversidade foram construídos com base nos dados de abundância das espécies lenhosas e herbáceas, dos tratamentos N (FOM) e $\mathrm{P}$ (Povoamento de pinus).

Para a comparação da riqueza nos tratamentos foram utilizadas curvas de interpolação/ extrapolação, esta última estimada para o dobro do esforço amostral. Ambas foram calculadas com base em dados de frequência das espécies. Os intervalos de confiança de $95 \%$ dos perfis de diversidade e as curvas de interpolação/ extrapolação foram calculados com base em 100 randomizações. As curvas de interpolação e extrapolação, bem como os perfis de diversidade, foram confeccionadas no programa iNEXT, com base em números de Hill (Chao 2016).

Foram realizadas análises ANOVA e teste Tukey para a comparação das médias de abundância e riqueza, bem como para a interação entre estas. Primeiramente foi ajustado um modelo linear e analisados a normalidade e homocedasticidade dos resíduos. Nos casos onde os resíduos não cumpriram com tais pressupostos, foram realizadas transformações Box-Cox fornecida pelo pacote MASS (Venables \& Ripley 2002). No caso de dados para os quais não foi atingido nenhum desses pressupostos, mesmo com transformação, foram ajustados modelos lineares generalizados com distribuição binomial negativa. As médias foram comparadas par a par por meio de contraste de Tukey, onde os $\mathrm{p}$-valores foram ajustados por meio de single-step (pacote multcomp, Hothorn et al. 2008). Estas análises foram realizadas com o auxílio dos programas estatísticos Assistat versão 7.7 pt 2, e R v. 3.2.5 (R Core Team 2016).

\section{Resultados}

Considerando todos os tratamentos, foi contabilizado um total de 11.669 indivíduos, distribuídos em 31 famílias botânicas, 66 gêneros e 84 espécies, das quais quatro são classificadas como espécies exóticas (Pinus taeda L., Hypochaeris radicata L., Sonchus asper (L.) Hill e Urochloa plantaginea (Link.) R.D.Webster.).

Do total de indivíduos identificados, 72,2\% (8.426 indivíduos) correspondem ao banco de sementes do povoamento de Pinus taeda, o qual apresentou densidades de 1566,80 e 118,40 sementes germinadas por $\mathrm{m}^{2}$ para indivíduos herbáceos e lenhosos, respectivamente. $\mathrm{Na}$ área do remanescente florestal essas densidades foram de 423,40 e 225,20 sementes germinadas por $\mathrm{m}^{2}$, respectivamente para herbáceas e lenhosas (Tab. 1).

Foi observada diferença significativa na abundância de indivíduos lenhosos $\left(\mathrm{F}_{(1,158)}=50,04\right.$; $\mathrm{P}<0,001)$ e herbáceos $\left(\mathrm{F}_{(1,158)}=201,54 ; \mathrm{P}<0,001\right)$ entre o banco de sementes das diferentes áreas. A riqueza entre as áreas mostrou-se diferente para espécies lenhosas $\left(\mathrm{F}_{(1,158)}=220,03 ; \mathrm{P}<0,001\right)$, mas não para as herbáceas $\left(\mathrm{F}_{(1,158)}=0,06 ; \mathrm{P}=0,79\right.$; Tab. 1).

No banco de sementes proveniente do remanescente florestal a distribuição do número de indivíduos germinados entre as formas de vida estabelecidas foi de $65,5 \%$ de indivíduos herbáceos e 34,5\% de indivíduos lenhosos. Já para o banco de sementes do povoamento de pinus houve maior disparidade, sendo que as espécies herbáceas abrangeram 93\% do número total de indivíduos daquela comunidade.

Os maiores valores de similaridade entre as áreas, pelo índice de Jaccard, foram para as espécies herbáceas $(0,45)$, seguida das lenhosas $(0,19)$. Quando consideradas ambas as formas de vida conjuntamente a similaridade entre as áreas foi de 0,34 .

As famílias que apresentaram maior riqueza de espécies herbáceas no banco de sementes da floresta nativa foram Asteraceae (13), Poaceae (7) e Solanaceae (4), tendo como espécies de maior representatividade estrutural Erechtites valerianifolius, Hypoxis decumbens, Phytolacca thyrsiflora, Coccocypselum sp. e Ichnanthus pallens, totalizando juntas quase $45 \%$ de percentual de importância (Tab. 2). A primeira e a terceira espécie destacaram-se especialmente pela sua maior frequência, tendo sido registradas em mais de $70 \%$ das amostras.

No povoamento de pinus as famílias de espécies herbáceas com maior representatividade foram Poaceae (8), Asteraceae (5) e Solanaceae (4). Apenas três espécies de Poaceae (Rugoloa pilosa, Panicum millegrana e Setaria scabrifolia) juntas somaram 51,3\% do percentual de importância, e $66 \%$ do total de indivíduos. Em termos de frequência destacaram-se $R$. pilosa e $S$. scabrifolia, que ocorreram em mais de $90 \%$ das amostras (Tab. 3).

Dentre as espécies lenhosas do banco de sementes da floresta nativa destacaram-se as famílias Solanaceae (10) e Asteraceae (5), onde as espécies Baccharis vulneraria, Solanum mauritianum e Mimosa scabrella apresentaram maior valor de importância, totalizando 56,6\% do 
Tabela 1 - Número de espécies, famílias e indivíduos do banco de sementes de um Remanescente de Floresta Ombrófila Mista (N) e de um Povoamento de Pinus (P) em Campo do Tenente, PR.

Table 1 - Number of species, families and individuals of the seed banks of a Remnant of Araucaria Rainforest (N) and of a Pine plantation (P) in Campo do Tenente, PR.

\begin{tabular}{lccccc}
\hline & Tratamento & Herbáceas & Herbáceas $/ \mathbf{m}^{2 *}$ & Lenhosa & Lenhosa/m $\mathbf{m}^{2 *}$ \\
\hline Espécies & $\mathrm{N}$ & 42 & 8,40 & 35 & 7 \\
& $\mathrm{P}$ & 28 & 5,60 & 8 & 1,60 \\
Famílias & $\mathrm{N}$ & 16 & 3,20 & 18 & 3,60 \\
& $\mathrm{P}$ & 10 & 2 & 5 & 1 \\
Indivíduos & $\mathrm{N}$ & 2117 & 423,40 & 1126 & 225,20 \\
& $\mathrm{P}$ & 7834 & 1566,80 & 592 & 118,40 \\
Riqueza de espécies média por & $\mathrm{N}$ & $7,03 \pm 2,12 \mathrm{a}$ & & $5,01 \pm 1,82 \mathrm{a}$ & \\
unidade amostral ( \pm DP) & $\mathrm{P}$ & $6,95 \pm 1,53 \mathrm{a}$ & & $1,68 \pm 0,88 \mathrm{~b}$ & \\
Abundância média por unidade & $\mathrm{N}$ & $26,46 \pm 24,69 \mathrm{a}$ & & $14,08 \pm 8,94 \mathrm{a}$ & \\
amostral $( \pm \mathrm{DP})$ & $\mathrm{P}$ & $97,93 \pm 63,03 \mathrm{~b}$ & & $7,40 \pm 7,43 \mathrm{~b}$ & \\
\hline
\end{tabular}

As médias seguidas pela mesma letra não diferem estatisticamente entre si pelo teste de Tukey $(\mathrm{P}<0.05)$.

* Valores calculados com base a área total amostrada para cada tratamento $\left(5 \mathrm{~m}^{2}\right)$.

Means followed by the same letter do not differ statistically from each other by the Tukey test $(\mathrm{P}<0.05)$.

* Values calculated based on the total area surveyed for each treatment $\left(5.00 \mathrm{~m}^{2}\right)$.

percentual de importância. As três espécies também se destacaram por ocorrer em mais de $70 \%$ das unidades amostrais (Tab. 4).

No banco de sementes do povoamento de pinus apenas as famílias Asteraceae (3) e Solanaceae (2) apresentaram mais de uma espécie. A espécie Baccharis vulneraria destacou-se com maior representatividade fitossociológica, seguida de Baccharis dracunculifolia, somando juntas quase $90 \%$ do percentual de importância desta comunidade. É válido mencionar que a espécie P. taeda apresentou apenas quatro indivíduos e, portanto, baixa frequência nas amostras, resultando consequentemente em reduzida importância fitossociológica, a despeito da abundância de matrizes adultas da espécie cobrindo toda a área do banco de sementes estudado (Tab. 5).

Os perfis de diversidade (Fig. 1a) denotam que no banco de sementes de floresta nativa existe uma maior diversidade de espécies herbáceas e lenhosas em comparação ao banco de sementes do povoamento de pinus. Para ambas as áreas as espécies herbáceas são mais diversas que as lenhosas, para quase todos os valores dos parâmetros q. Apenas a riqueza total de espécies $(\mathrm{q}=0)$ germinadas na floresta nativa nas diferentes formas de vida não apresentou diferença significativa, considerando a avaliação total.
As curvas de interpolação/extrapolação apontam que houve diferença significativa entre as espécies lenhosas do banco de sementes do povoamento de pinus e as demais, em todas as unidades amostrais (Fig. 1b).

A maior diversidade do banco de sementes da floresta nativa resultou em curvas de interpolação/ extrapolação onde se verifica que um número maior de amostras seria necessário para abranger a amplitude florística da comunidade, sendo que um aumento de $100 \%$ da amostragem resultaria em aumentos de 40 a $50 \%$ do número de espécies. No povoamento de pinus o número amostral foi suficiente para ambas as formas de vida, sendo que o aumento de $100 \%$ na amostragem repercutiria em menos de $15 \%$ de aumento do número de espécies (Fig. 1b).

\section{Discussão}

A riqueza de espécies lenhosas e herbáceas registrada no banco de sementes do remanescente de floresta nativa foi bastante expressiva quando comparada a de outros bancos de sementes de Floresta Ombrófila Mista (Chami et al. 2011; Souza et al. 2011; Schorn et al. 2013), onde a riqueza em geral, considerando herbáceas e lenhosas, não ultrapassou 60 espécies e 22 famílias, considerando 
Tabela 2 - Parâmetros fitossociológicos das espécies herbáceas do banco de sementes de um remanescente de Floresta Ombrófila Mista em Campo do Tenente, PR. Os cinco maiores percentuais de importância foram assinalados em cinza. $\mathrm{N}^{o}$ ind = número de indivíduos; Dens Abs = densidade absoluta; Dens Rel = densidade relativa; Freq Abs = frequência absoluta; Freq Rel = frequência relativa; Valor Imp = valor de importância.

Table 2 - Phytosociological parameters of the herbaceous species of the seed bank of a remnant of Araucaria Rainforest in Campo do Tenente, PR. The five highest percentages of importance were marked in gray. $\mathrm{N}^{\circ}$ ind = number of individuals; Dens Abs $=$ absolute density; Dens Rel = relative density; Freq Abs = absolute frequency; Rel Frequency = relative frequency; Valor Imp = value of importance.

\begin{tabular}{|c|c|c|c|c|c|c|}
\hline Família / Espécie & $\mathbf{N}^{0}$ ind & $\begin{array}{l}\text { Dens Abs } \\
\left(\mathbf{s e m} / \mathbf{m}^{2}\right)\end{array}$ & $\begin{array}{c}\text { Dens Rel } \\
(\%)\end{array}$ & $\begin{array}{c}\text { Freq Abs } \\
(\%)\end{array}$ & $\begin{array}{c}\text { Freq Rel } \\
(\%)\end{array}$ & $\begin{array}{c}\text { Valor Imp } \\
(\%)\end{array}$ \\
\hline \multicolumn{7}{|l|}{ Asteraceae } \\
\hline Ageratum conyzoides $\mathrm{L}$. & 1 & 0,2 & 0,05 & 1,25 & 0,18 & 0,11 \\
\hline Ambrosia polystachya DC. & 26 & 5,2 & 1,23 & 18,75 & 2,67 & 1,95 \\
\hline Baccharis sp. & 1 & 0,2 & 0,05 & 1,25 & 0,18 & 0,11 \\
\hline Chaptalia nutans (L.) Polak & 1 & 0,2 & 0,05 & 1,25 & 0,18 & 0,11 \\
\hline Conyza bonariensis (L.) Cronquist & 11 & 2,2 & 0,52 & 11,25 & 1,60 & 1,06 \\
\hline Erechtites hieracifolius (L.) Raf. ex DC. & 2 & 0,4 & 0,09 & 2,5 & 0,36 & 0,23 \\
\hline Erechtites valerianifolius (Link ex Spreng.) DC. & 195 & 39 & 9,21 & 83,75 & 11,92 & 10,57 \\
\hline Gamochaeta pensylvanica (Willd.) Cabrera & 110 & 22 & 5,20 & 45 & 6,41 & 5,80 \\
\hline Gamochaeta purpurea (L.) Cabrera & 45 & 9 & 2,13 & 23,75 & 3,38 & 2,75 \\
\hline Hypochaeris chillensis (Kunth) Britton & 1 & 0,2 & 0,05 & 1,25 & 0,18 & 0,11 \\
\hline Hypochaeris radicata $\mathrm{L} . *$ & 1 & 0,2 & 0,05 & 1,25 & 0,18 & 0,11 \\
\hline Senecio brasiliensis (Spreng.) Less. & 1 & 0,2 & 0,05 & 1,25 & 0,18 & 0,11 \\
\hline Sonchus asper (L.) Hill* & 3 & 0,6 & 0,14 & 3,75 & 0,53 & 0,34 \\
\hline \multicolumn{7}{|l|}{ Begoniaceae } \\
\hline Begonia cucullata Willd. \& Otto & 3 & 0,6 & 0,14 & 3,75 & 0,53 & 0,34 \\
\hline \multicolumn{7}{|l|}{ Convolvulaceae } \\
\hline Ipomoea sp. & 3 & 0,6 & 0,14 & 3,75 & 0,53 & 0,34 \\
\hline \multicolumn{7}{|l|}{ Cyperaceae } \\
\hline Bulbostylis capillaris (L.) C.B. Clarke & 1 & 0,2 & 0,05 & 1,25 & 0,18 & 0,11 \\
\hline Cyperus meyenianus Kunth. & 2 & 0,4 & 0,09 & 2,5 & 0,36 & 0,23 \\
\hline Rhynchospora corymbosa (L.) Britton & 24 & 4,8 & 1,13 & 8,75 & 1,25 & 1,19 \\
\hline \multicolumn{7}{|l|}{ Hypoxidaceae } \\
\hline Hypoxis decumbens $\mathrm{L}$. & 269 & 53,8 & 12,71 & 58,75 & 8,36 & 10,53 \\
\hline \multicolumn{7}{|l|}{ Iridaceae } \\
\hline Sisyrinchium vaginatum Sprengel. & 5 & 1 & 0,24 & 5 & 0,71 & 0,47 \\
\hline \multicolumn{7}{|l|}{ Malvaceae } \\
\hline Pavonia sp. & 3 & 0,6 & 0,14 & 1,25 & 0,18 & 0,16 \\
\hline Sida rhombifolia L. & 2 & 0,4 & 0,09 & 2,5 & 0,36 & 0,23 \\
\hline Wissadula parviflora (A.St.-Hil.) R.E.Fries & 16 & 3,2 & 0,76 & 13,75 & 1,96 & 1,36 \\
\hline \multicolumn{7}{|l|}{ Melastomataceae } \\
\hline Chaetogastra clinopodifolia Cogn. & 2 & 0,4 & 0,09 & 1,25 & 0,18 & 0,14 \\
\hline \multicolumn{7}{|l|}{ Phytolaccaceae } \\
\hline Phytolacca thyrsiflora Fenzl ex J.A.Schimidt. & 149 & 29,8 & 7,04 & 71,25 & 10,14 & 8,59 \\
\hline
\end{tabular}




\begin{tabular}{|c|c|c|c|c|c|c|}
\hline Família / Espécie & $\mathbf{N}^{0}$ ind & $\begin{array}{c}\text { Dens Abs } \\
\left(\mathrm{sem} / \mathbf{m}^{2}\right)\end{array}$ & $\begin{array}{c}\text { Dens Rel } \\
(\%)\end{array}$ & $\begin{array}{l}\text { Freq Abs } \\
\quad(\%)\end{array}$ & $\begin{array}{c}\text { Freq Rel } \\
(\%)\end{array}$ & $\begin{array}{c}\text { Valor Imp } \\
(\%)\end{array}$ \\
\hline \multicolumn{7}{|l|}{ Plantaginaceae } \\
\hline Plantago guilleminiana Decne. & 1 & 0,2 & 0,05 & 1,25 & 0,18 & 0,11 \\
\hline \multicolumn{7}{|l|}{ Poaceae } \\
\hline Dichanthelium stigmosum (Trin.) Zuloaga & 123 & 24,6 & 5,81 & 26,25 & 3,74 & 4,77 \\
\hline Ichnanthus pallens (Sw.) Munro ex Benth. & 203 & 40,6 & 9,59 & 35 & 4,98 & 7,29 \\
\hline Panicum millegrana Poir. & 34 & 6,8 & 1,61 & 6,25 & 0,89 & 1,25 \\
\hline Paspalum paniculatum $\mathrm{L}$. & 10 & 2 & 0,47 & 2,5 & 0,36 & 0,41 \\
\hline Rugoloa pilosa (Sw.) Zuloaga & 143 & 28,6 & 6,75 & 31,25 & 4,45 & 5,60 \\
\hline Setaria scabrifolia (Nees) Kunth & 253 & 50,6 & 11,95 & 8,75 & 1,25 & 6,60 \\
\hline Urochloa plantaginea (Link.) R.D.Webster.* & 12 & 2,4 & 0,57 & 12,5 & 1,78 & 1,17 \\
\hline \multicolumn{7}{|l|}{ Rubiaceae } \\
\hline Coccocypselum sp. & 188 & 37,6 & 8,88 & 47,5 & 6,76 & 7,82 \\
\hline Coccocypselum lanceolatum (Ruiz \& Pav.) Per. & 9 & 1,8 & 0,43 & 3,75 & 0,53 & 0,48 \\
\hline Mitracarpus hirtus (L.) DC & 24 & 4,8 & 1,13 & 13,75 & 1,96 & 1,55 \\
\hline \multicolumn{7}{|l|}{ Solanaceae } \\
\hline Nicotiana langsdorffii Weinmann & 24 & 4,8 & 1,13 & 18,75 & 2,67 & 1,90 \\
\hline Petunia scheideana L.B.Sm. \& Downs & 3 & 0,6 & 0,14 & 3,75 & 0,53 & 0,34 \\
\hline Solanum americanum Mill. & 98 & 19,6 & 4,63 & 62,5 & 8,90 & 6,76 \\
\hline Solanum sisymbriifolium Lam. & 101 & 20,2 & 4,77 & 53,75 & 7,65 & 6,21 \\
\hline \multicolumn{7}{|l|}{ Violaceae } \\
\hline Pombalia parviflora (Mutis ex L.f.) Paula-Souza & 13 & 2,6 & 0,61 & 3,75 & 0,53 & 0,57 \\
\hline Viola cerasifolia A.St.-Hil. & 1 & 0,2 & 0,05 & 1,25 & 0,18 & 0,11 \\
\hline Total & 2.117 & 423,40 & 100 & 702,50 & 100 & 100 \\
\hline
\end{tabular}

que todas as avaliações apresentaram área amostral semelhante. Apenas no estudo de Silva-Weber et al. (2012), em FOM Aluvial, o montante de espécies inventariadas foi superior, entretanto a área amostral é também superior ao avaliado neste estudo.

No banco de sementes do povoamento de Pinus taeda a riqueza foi significativamente menor à encontrada na floresta nativa, sobretudo no estrato das espécies lenhosas. Este resultado é corroborado por outros estudos que avaliaram bancos de sementes de áreas cobertas por povoamentos do gênero Pinus, nos quais a diversidade florística foi igualmente reduzida (Gonçalves et al. 2008; Schorn et al. 2013).

Por outro lado, o número médio de plântulas (de lenhosas e herbáceas) germinadas no solo do povoamento de pinus foi cerca de duas vezes maior do que da floresta nativa, essencialmente devido à grande densidade de indivíduos herbáceos. Esta relação entre o número de plântulas emergidas por unidade de área é relativa ao ambiente avaliado, bem como ao uso e a ocupação do solo da área em avaliação (Schorn et al. 2013).

A proporção entre a densidade de espécies lenhosas e herbáceas no banco de sementes da floresta nativa foi equivalente ao registrado em uma Floresta Ombrófila Densa Montana no Parque Estadual Intervales, São Paulo (Baider et al. 1999). Já o expressivo predomínio de indivíduos herbáceos em relação aos lenhosos no povoamento de pinus é congruente com uma tendência verificada também em outras áreas perturbadas (Garwood 1989; Costalonga et al. 2006; Schorn et 
Tabela 3 - Parâmetros fitossociológicos das espécies herbáceas do banco de sementes de um povoamento de Pinus taeda em Campo do Tenente, PR. Os três maiores percentuais de importância foram assinalados em cinza. $\mathrm{N}^{\circ}$ ind = número de indivíduos; Dens Abs = densidade absoluta; Dens Rel = densidade relativa; Freq Abs = frequência absoluta; Freq Rel = frequência relativa; Valor Imp = valor de importância.

Table 3 - Phytosociological parameters of the herbaceous species of the seed bank of a pine plantation in Campo do Tenente, PR. The three highest percentages of importance were marked in gray. $\mathrm{N}^{\mathrm{o}}$ ind $=$ number of individuals; Dens Abs = absolute density; Dens Rel $=$ relative density; Freq Abs = absolute frequency; Rel Frequency = relative frequency; Imp Value = value of importance.

\begin{tabular}{|c|c|c|c|c|c|c|}
\hline Família / Espécie & $\mathrm{N}^{0}$ ind & $\begin{array}{l}\text { Dens Abs } \\
\left(\mathbf{s e m} / \mathbf{m}^{2}\right)\end{array}$ & $\begin{array}{l}\text { Dens Rel } \\
(\%)\end{array}$ & $\begin{array}{l}\text { Freq Abs } \\
\quad(\%)\end{array}$ & $\begin{array}{l}\text { Freq Rel } \\
(\%)\end{array}$ & $\begin{array}{l}\text { Valor Imp } \\
\quad(\%)\end{array}$ \\
\hline \multicolumn{7}{|l|}{ Asteraceae } \\
\hline $\begin{array}{l}\text { Austroeupatorium laetevirens (Hook. \& Arn.) } \\
\text { R.M. King \& H. Rob. }\end{array}$ & 33 & 6,60 & 0,42 & 16,25 & 2,33 & 1,38 \\
\hline Erechtites hieracifolius (L.) Raf. ex DC. & 5 & 1 & 0,06 & 6,25 & 0,90 & 0,48 \\
\hline Erechtites valerianifolius (Link ex Spreng.) DC. & 52 & 10,40 & 0,66 & 36,25 & 5,21 & 2,94 \\
\hline Gamochaeta pensylvanica (Willd.) Cabrera & 69 & 13,80 & 0,88 & 18,75 & 2,69 & 1,79 \\
\hline Gamochaeta purpurea (L.) Cabrera & 104 & 20,80 & 1,33 & 42,50 & 6,10 & 3,72 \\
\hline \multicolumn{7}{|l|}{ Commelinaceae } \\
\hline Commelina obliqua Vahl. & 15 & 3 & 0,19 & 8,75 & 1,26 & 0,72 \\
\hline \multicolumn{7}{|l|}{ Cyperaceae } \\
\hline Bulbostylis capillaris (L.) C.B. Clarke & 393 & 78,60 & 5,02 & 38,75 & 5,57 & 5,29 \\
\hline Cyperus meyenianus Kunth. & 15 & 3 & 0,19 & 2,50 & 0,36 & 0,28 \\
\hline Rhynchospora corymbosa (L.) Britton & 11 & 2,20 & 0,14 & 6,25 & 0,90 & 0,52 \\
\hline \multicolumn{7}{|l|}{ Iridaceae } \\
\hline Sisyrinchium vaginatum Sprengel. & 35 & 7 & 0,45 & 7,50 & 1,08 & 0,76 \\
\hline \multicolumn{7}{|l|}{ Phyllanthaceae } \\
\hline Phyllanthus niruri $\mathrm{L}$. & 43 & 8,60 & 0,55 & 11,25 & 1,62 & 1,08 \\
\hline \multicolumn{7}{|l|}{ Phytolaccaceae } \\
\hline Phytolacca thyrsiflora Fenzl ex J.A.Schimidt. & 5 & 1 & 0,06 & 6,25 & 0,90 & 0,48 \\
\hline \multicolumn{7}{|l|}{ Poaceae } \\
\hline Dichanthelium stigmosum (Trin.) Zuloaga & 7 & 1,40 & 0,09 & 2,50 & 0,36 & 0,22 \\
\hline Ichnanthus pallens (Sw.) Munro ex Benth. & 251 & 50,20 & 3,20 & 47,50 & 6,82 & 5,01 \\
\hline Panicum millegrana Poir. & 1411 & 282,20 & 18,01 & 65 & 9,34 & 13,67 \\
\hline Paspalum paniculatum $\mathrm{L}$. & 502 & 100,40 & 6,41 & 50 & 7,18 & 6,79 \\
\hline Rugoloa pilosa (Sw.) Zuloaga & 2880 & 576 & 36,76 & 95 & 13,64 & 25,20 \\
\hline Setaria scabrifolia (Nees) Kunth & 894 & 178,80 & 11,41 & 93,75 & 13,46 & 12,44 \\
\hline Steinchisma laxum (Sw.) Zuloaga & 17 & 3,40 & 0,22 & 8,75 & 1,26 & 0,74 \\
\hline Urochloa plantaginea (Link.) R.D. Webster* & 1 & 0,20 & 0,01 & 1,25 & 0,18 & 0,10 \\
\hline \multicolumn{7}{|l|}{ Rubiaceae } \\
\hline Mitracarpus hitrus (L.) DC & 259 & 51,80 & 3,31 & 41,25 & 5,92 & 4,62 \\
\hline Richardia brasiliensis (Cham e Schltdl.) Steud. & 788 & 157,60 & 10,06 & 61,25 & 8,80 & 9,43 \\
\hline \multicolumn{7}{|l|}{ Solanaceae } \\
\hline Calibrachoa excellens (R.E.Fr.) Wijsman & 13 & 2,60 & 0,17 & 3,75 & 0,54 & 0,35 \\
\hline
\end{tabular}




\begin{tabular}{lcccccc}
\hline Família / Espécie & $\mathbf{N}^{\mathbf{0}}$ ind & $\begin{array}{c}\text { Dens Abs } \\
\left(\mathbf{s e m} / \mathbf{m}^{\mathbf{2}}\right.\end{array}$ & $\begin{array}{c}\text { Dens Rel } \\
\mathbf{( \% )}\end{array}$ & $\begin{array}{c}\text { Freq Abs } \\
\mathbf{( \% )}\end{array}$ & $\begin{array}{c}\text { Freq Rel } \\
\mathbf{( \% )}\end{array}$ & $\begin{array}{c}\text { Valor Imp } \\
\mathbf{( \% )}\end{array}$ \\
\hline Petunia scheideana L.B.Sm. \& Downs & 1 & 0,20 & 0,01 & 1,25 & 0,18 & 0,10 \\
Solanum americanum Mill. & 9 & 1,80 & 0,11 & 10 & 1,44 & 0,78 \\
$\begin{array}{l}\text { Solanum sisymbriifolium Lam. } \\
\text { Violaceae }\end{array}$ & 7 & 1,40 & 0,09 & 3,75 & 0,54 & 0,31 \\
$\begin{array}{l}\text { Pombalia parviflora (Mutis ex L.f.) Paula-Souza } \\
\text { Viola cerasifolia A.St.-Hil. }\end{array}$ & 12 & 2,40 & 0,15 & 7,50 & 1,08 & 0,62 \\
\hline Total & 2 & 0,40 & 0,03 & 2,50 & 0,36 & 0,19 \\
\hline
\end{tabular}

* Espécie exótica invasora

* Invasive exotic species

al. 2013). O alto número de indivíduos herbáceos no banco de sementes pode ser justificado uma vez que em geral estas são espécies ruderais cujas sementes apresentam viabilidade por períodos maiores (Gasparino et al. 2006; Gonçalves et al. 2008), além de manifestar intensa capacidade de estabelecimento e crescimento rápido das plântulas em ambientes altamente perturbados (Grime 1974).

A diferença significativa na abundância de indivíduos herbáceos entre as duas áreas avaliadas tem como justificativa a tendência destas espécies predominarem em ambientes perturbados, sendo, portanto, favorecidas pelos distúrbios relacionados ao manejo do povoamento de pinus, que propiciam maior entrada de sementes (Baider et al. 2001; Araújo et al. 2004). Deve-se ainda considerar que as sementes de espécies herbáceas em geral apresentam facilidade de entrada e incorporação no solo dado ao seu reduzido tamanho, além de suportar condições adversas e serem pouco exigentes quanto às condições edáficas (Siqueira 2002; Vinha 2008).

Confirmando a tendência verificada no presente estudo, a riqueza de espécies lenhosas no banco de sementes de povoamentos homogêneos de espécies exóticas é reduzida em relação às outras formas de vida observadas, quando comparada à riqueza de lenhosas em bancos de sementes de remanescentes florestais nativos (Calegari et al. 2013; Schorn et al. 2013), aspecto que demonstra a resistência do povoamento monoespecífico quanto à entrada e formação de estoque de sementes de espécies lenhosas. Em povoamentos do gênero Pinus, esta limitação pode estar associada à elevada densidade do dossel, o qual dificulta a chegada das sementes ao solo(Schorn et al. 2013). A redução de diversidade de espécies pode ainda estar relacionada à densa camada de acículas acumulada no solo, como apontado em condições semelhantes (Abreu 2013). Os fatores citados parecem não afetar do mesmo modo as espécies herbáceas neste estudo as quais, pela sua grande adaptabilidade e baixa exigência edáfica, tendem a apresentar maior número de espécies e indivíduos nestes ambientes (Siqueira 2002; Vinha 2008).

Das espécies que apresentaram maior valor de importância no banco de sementes do remanescente florestal nativo, Ichnanthus pallens e Hypoxis decumbens aparecem igualmente entre as de maior relevância em um estudo que avalia apenas a sinúsia herbácea adulta de floresta com araucária (CitadiniZanette et al. 2011). Todas as espécies herbáceas de maior percentual de importância aparecem ainda em outros estudos, dentro da mesma formação florestal, no entanto com menor relevância na comunidade (Kozera et al. 2006; Souza et al. 2011; Schorn et al. 2013). Entre espécies lenhosas, os principais táxons amostrados não fazem correspondência com resultados de trabalhos realizados na mesma unidade fitogeográfica, apresentando grande variabilidade entre as espécies que aparecem com maior percentual de importância entre remanescentes florestais distintos (Caldato et al. 1996; Avila et al. 2011; Chami et al. 2011; Schorn et al. 2013).

A diferença na composição florística entre os dois bancos de sementes avaliados foi também observada, porém de modo menos expressivo, em estudo análogo entre um povoamento de pinus e um fragmento de FOM em Rio Negrinho, Santa Catarina (Schorn et al. 2013). No referido estudo, quando confrontadas as principais espécies herbáceas amostradas entre o povoamento de pinus avaliado com o banco de sementes de um 
Tabela 4 - Parâmetros fitossociológicos das espécies lenhosas do banco de sementes de um remanescente de Floresta Ombrófila Mista em Campo do Tenente, PR. Os três maiores percentuais de importância foram assinalados em cinza. $\mathrm{N}^{\mathrm{o}}$ ind = número de indivíduos; Dens Abs = densidade absoluta; Dens Rel = densidade relativa; Freq Abs = frequência absoluta; Freq Rel = frequência relativa; Valor Imp = valor de importância.

Table 4 - Phytosociological parameters of the woody species of the seed bank of a remnant of Araucaria Rainforest in Campo do Tenente, PR. The three highest percentages of importance were marked in gray. $\mathrm{N}^{\circ}$ ind = number of individuals; Dens Abs = absolute density; Dens Rel = relative density; Freq Abs = absolute frequency; Rel Frequency = relative frequency; Valor Imp = value of importance.

\begin{tabular}{|c|c|c|c|c|c|c|}
\hline Família / Espécie & $\mathbf{N}^{o}$ ind & $\begin{array}{c}\text { Dens Abs } \\
\left(\mathbf{s e m} / \mathbf{m}^{2}\right)\end{array}$ & $\begin{array}{c}\text { Dens Rel } \\
(\%)\end{array}$ & $\begin{array}{c}\text { Freq Abs } \\
(\%)\end{array}$ & $\begin{array}{c}\text { Freq Rel } \\
(\%)\end{array}$ & $\begin{array}{c}\text { Valor Imp } \\
(\%)\end{array}$ \\
\hline \multicolumn{7}{|l|}{ Annonaceae } \\
\hline Annona rugulosa (Schltdl.) H. Rainer & 1 & 0,20 & 0,09 & 1,25 & 0,25 & 0,17 \\
\hline \multicolumn{7}{|l|}{ Apocynaceae } \\
\hline Apocynaceae sp. & 2 & 0,40 & 0,18 & 2,50 & 0,50 & 0,34 \\
\hline \multicolumn{7}{|l|}{ Aquifoliaceae } \\
\hline Ilex brevicuspis Reissek & 5 & 1 & 0,44 & 1,25 & 0,25 & 0,35 \\
\hline \multicolumn{7}{|l|}{ Asparagaceae } \\
\hline Cordyline spectabilis Kunth \& Bouché & 14 & 2,80 & 1,24 & 1,25 & 0,25 & 0,75 \\
\hline \multicolumn{7}{|l|}{ Asteraceae } \\
\hline Asteraceae sp. 01 & 1 & 0,20 & 0,09 & 1,25 & 0,25 & 0,17 \\
\hline Asteraceae sp. 02 & 1 & 0,20 & 0,09 & 1,25 & 0,25 & 0,17 \\
\hline Asteraceae sp. 03 & 32 & 6,40 & 2,84 & 26,25 & 5,24 & 4,04 \\
\hline Baccharis dracunculifolia DC. & 14 & 2,80 & 1,24 & 15 & 2,99 & 2,12 \\
\hline Baccharis vulneraria Baker & 200 & 40 & 17,76 & 83,75 & 16,71 & 17,24 \\
\hline \multicolumn{7}{|l|}{ Bignoniaceae } \\
\hline Jacaranda puberula Cham. & 7 & 1,40 & 0,62 & 7,50 & 1,50 & 1,06 \\
\hline \multicolumn{7}{|l|}{ Cannabaceae } \\
\hline Trema micrantha (L.) Blume & 28 & 5,60 & 2,49 & 22,50 & 4,49 & 3,49 \\
\hline \multicolumn{7}{|l|}{ Clethraceae } \\
\hline Clethra scabra Pers. & 58 & 11,60 & 5,15 & 17,50 & 3,49 & 4,32 \\
\hline \multicolumn{7}{|l|}{ Euphorbiaceae } \\
\hline Sapium glandulosum (L.) Morong & 3 & 0,60 & 0,27 & 3,75 & 0,75 & 0,51 \\
\hline \multicolumn{7}{|l|}{ Fabaceae } \\
\hline Mimosa scabrella Benth. & 177 & 35,40 & 15,72 & 73,75 & 14,71 & 15,22 \\
\hline \multicolumn{7}{|l|}{ Hypericaceae } \\
\hline Hypericum brasiliense Choisy & 61 & 12,20 & 5,42 & 26,25 & 5,24 & 5,33 \\
\hline \multicolumn{7}{|l|}{ Lauraceae } \\
\hline Ocotea puberula (Rich.) Ness & 16 & 3,20 & 1,42 & 16,25 & 3,24 & 2,33 \\
\hline \multicolumn{7}{|l|}{ Primulaceae } \\
\hline Myrsine coriacea (Sw.) R. Br. & 3 & 0,60 & 0,27 & 2,50 & 0,50 & 0,38 \\
\hline Myrsine umbellata Mart. & 8 & 1,60 & 0,71 & 10 & 2 & 1,35 \\
\hline \multicolumn{7}{|l|}{ Rubiaceae } \\
\hline Psychotria leiocarpa Cham. \& Schltdl. & 1 & 0,20 & 0,09 & 1,25 & 0,25 & 0,17 \\
\hline
\end{tabular}




\begin{tabular}{|c|c|c|c|c|c|c|}
\hline Família / Espécie & $\mathrm{N}^{0}$ ind & $\begin{array}{c}\text { Dens Abs } \\
\left(\mathbf{s e m} / \mathbf{m}^{2}\right)\end{array}$ & $\begin{array}{c}\text { Dens Rel } \\
(\%)\end{array}$ & $\begin{array}{c}\text { Freq Abs } \\
(\%)\end{array}$ & $\begin{array}{c}\text { Freq Rel } \\
(\%)\end{array}$ & $\begin{array}{c}\text { Valor Imp } \\
(\%)\end{array}$ \\
\hline Palicourea marcgravii St. Hil. & 3 & 0,60 & 0,27 & 1,25 & 0,25 & 0,26 \\
\hline \multicolumn{7}{|l|}{ Rutaceae } \\
\hline Zanthoxylum rhoifolium Lam. & 29 & 5,80 & 2,58 & 16,25 & 3,24 & 2,91 \\
\hline \multicolumn{7}{|l|}{ Salicaceae } \\
\hline Casearia decandra Jacq. & 4 & 0,80 & 0,36 & 2,50 & 0,50 & 0,43 \\
\hline Casearia obliqua Spreng. & 7 & 1,40 & 0,62 & 5 & 1 & 0,81 \\
\hline Casearia sylvestris $\mathrm{Sw}$. & 83 & 16,60 & 7,37 & 47,50 & 9,48 & 8,42 \\
\hline \multicolumn{7}{|l|}{ Solanaceae } \\
\hline Solanum sp. 02 & 2 & 0,40 & 0,18 & 2,50 & 0,50 & 0,34 \\
\hline Solanum sp. 03 & 3 & 0,60 & 0,27 & 3,75 & 0,75 & 0,51 \\
\hline Solanum sp. 04 & 3 & 0,60 & 0,27 & 3,75 & 0,75 & 0,51 \\
\hline Solanum sp. 05 & 2 & 0,40 & 0,18 & 2,50 & 0,50 & 0,34 \\
\hline Solanum sp. 06 & 2 & 0,40 & 0,18 & 1,25 & 0,25 & 0,21 \\
\hline Solanum sp. 07 & 4 & 0,80 & 0,36 & 5 & 1 & 0,68 \\
\hline Solanum mauritianum Scop. & 345 & 69 & 30,64 & 88,75 & 17,71 & 24,17 \\
\hline Solanum pseudoquina A. St.-Hil. & 1 & 0,20 & 0,09 & 1,25 & 0,25 & 0,17 \\
\hline Solanum sanctaecatharinae Dunal & 4 & 0,80 & 0,36 & 2,50 & 0,50 & 0,43 \\
\hline Não identificada & 1 & 0,20 & 0,09 & 1,25 & 0,25 & 0,17 \\
\hline \multicolumn{7}{|l|}{ Família NI } \\
\hline Não Identificada & 1 & 0,20 & 0,09 & 1,25 & 0,25 & 0,17 \\
\hline Total & 1.126 & 225,20 & 100 & 501,25 & 100 & 100 \\
\hline
\end{tabular}

plantio equivalente, nenhuma das espécies de maior importância teve correspondência (Schorn et al. 2013).

Das quatro espécies herbáceas predominantes observadas no banco de sementes do povoamento de pinus, três são da família Poaceae, a qual é apresentada na literatura por possuir grande potencial de dispersão e a quarta espécie, também no povoamento de pinus, Richardia brasiliensis (Rubiaceae), também se prolifera rapidamente nos mais variados meios, entretanto, esta apresenta ainda intenso vigor vegetativo podendo ocupar completamente o solo de determinadas áreas (Lorenzi 2008; Souza \& Lorenzi 2008).

Assim como observado no presente estudo, em avaliações similares, embora em diferentes formações florestais, exemplares da espécie exótica em questão emergiram exclusivamente nos bancos de sementes dos próprios plantios puros (Calegari et al. 2013; Schorn et al. 2013). De um modo geral, as formações florestais são menos suscetíveis à invasão pelo gênero Pinus (Richardson \& Bond 1991). Este fato justifica-se, sobretudo, pelo gênero Pinus ser constituído essencialmente por espécies pioneiras, as quais se desenvolvem melhor em áreas abertas, onde a incidência de luz solar é maior (Zanchetta \& Diniz 2006), o que torna a germinação e crescimento destas espécies mais favoráveis (Tabarelli \& Mantovani 1999).

A baixa ocorrência de indivíduos de $P$. taeda germinados a partir do banco de sementes do povoamento de pinus deve-se possivelmente ao fato da serapilheira não ter sido considerada neste experimento. Considerando seu tamanho reduzido, as sementes podem ter encontrado na própria serapilheira uma barreira física e assim não alcançando o solo (Vargas \& Bernardi 2003). Este fator justifica também a reduzida entrada de outras espécies lenhosas no banco de sementes do povoamento de pinus.

Embora as sementes de $P$. taeda apresentem dormência (Cézar 2011), após o início da dispersão 
Tabela 5 - Parâmetros fitossociológicos das espécies lenhosas do banco de sementes de um povoamento de Pinus taeda em Campo do Tenente, PR. Os dois maiores percentuais de importância foram assinalados em cinza. $\mathrm{N}^{\mathrm{o}}$ ind = número de indivíduos; Dens Abs = densidade absoluta; Dens Rel = densidade relativa; Freq Abs = frequência absoluta; Freq Rel = frequência relativa; Valor Imp = valor de importância.

Table 5 - Phytosociological parameters of the woody species of the seed bank of a pine plantation in Campo do Tenente, PR. The two highest percentages of importance were marked in gray. $\mathrm{N}^{\mathrm{o}}$ ind $=$ number of individuals; Dens Abs = absolute density; Dens Rel = relative density; Freq Abs = absolute frequency; Rel Frequency = relative frequency; Imp Value = value of importance.

\begin{tabular}{|c|c|c|c|c|c|c|}
\hline Família / Espécie & $\mathbf{N}^{0}$ ind & $\begin{array}{c}\text { Dens Abs } \\
\left(\mathrm{sem} / \mathbf{m}^{2}\right)\end{array}$ & $\begin{array}{c}\text { Dens Rel } \\
(\%)\end{array}$ & $\begin{array}{c}\text { Freq Abs } \\
(\%)\end{array}$ & $\begin{array}{c}\text { Freq Rel } \\
(\%)\end{array}$ & $\begin{array}{c}\text { Valor Imp } \\
(\%)\end{array}$ \\
\hline \multicolumn{7}{|l|}{ Asteraceae } \\
\hline Asteraceae sp. 03 & 6 & 1,20 & 1,01 & 3,75 & 2,24 & 1,63 \\
\hline Baccharis dracunculifolia DC. & 120 & 24 & 20,27 & 52,50 & 31,34 & 25,81 \\
\hline Baccharis vulneraria Baker & 432 & 86,40 & 72,97 & 87,50 & 52,24 & 62,61 \\
\hline \multicolumn{7}{|l|}{ Hypericaceae } \\
\hline Hypericum brasiliense Choisy & 23 & 4,60 & 3,89 & 10 & 5,97 & 4,93 \\
\hline \multicolumn{7}{|l|}{ Pinaceae } \\
\hline Pinus taeda L.* & 4 & 0,80 & 0,68 & 5 & 2,99 & 1,83 \\
\hline \multicolumn{7}{|l|}{ Primulaceae } \\
\hline Myrsine coriacea (Sw.) R. Br. ex Roem \& Schult. & 1 & 0,20 & 0,17 & 1,25 & 0,75 & 0,46 \\
\hline \multicolumn{7}{|l|}{ Solanaceae } \\
\hline Solanum sp. 07 & 4 & 0,80 & 0,68 & 5 & 2,99 & 1,83 \\
\hline Solanum mauritianum Scop. & 2 & 0,40 & 0,34 & 2,50 & 1,49 & 0,92 \\
\hline Total & 592 & 118,40 & 100 & 167,50 & 100 & 100 \\
\hline
\end{tabular}

das sementes a capacidade germinativa destas é alta já nos primeiros seis meses, sendo que após este período o percentual germinativo cai para aproximadamente 50\% (Jankovski 1996). Desta forma, dado o período de realização do experimento é possível que a baixa incidência de exemplares de pinus esteja de fato mais associada ao acúmulo de serapilheira, ainda que os efeitos da dormência não devam ser descartados.

Por fim, quanto à suficiência amostral, observada pela estabilidade das curvas de interpolação, esta foi alcançada em todos os tratamentos, sobretudo na área do povoamento de pinus. No entanto, estima-se que um maior número de parcelas promoveria um aumento no número de espécies herbáceas e lenhosas germinadas no remanescente de floresta nativa, podendo-se em trabalhos futuros correlatos aumentar o número de parcelas para 160 unidades amostrais, com as quais, baseado no intervalo de confiança, pode-se atingir aumento de até 50\% na riqueza de espécies. A homogeneidade dos ambientes avaliados é de grande relevância, uma vez que a estabilização da curva amostral é atingida mais rapidamente quanto menor a variação florística entre as parcelas (Kersten \& Galvão 2013).

\section{Conclusão}

O povoamento de Pinus taeda, de 15 anos em área a 30 anos de cultivo, interfere na composição do banco de sementes em região de Floresta Ombrófila Mista, causando redução significativa de riqueza e densidade de espécies lenhosas.

O banco de sementes do remanescente de Floresta Ombrófila Mista em estágio médio de sucessão não sofreu invasão pela presença de povoamentos de Pinus taeda com aproximadamente $1 \mathrm{~km}$ de distância, demonstrando que esta distância pode ser uma barreira ao ingresso da espécie exótica invasora no remanescente florestal nativo. 

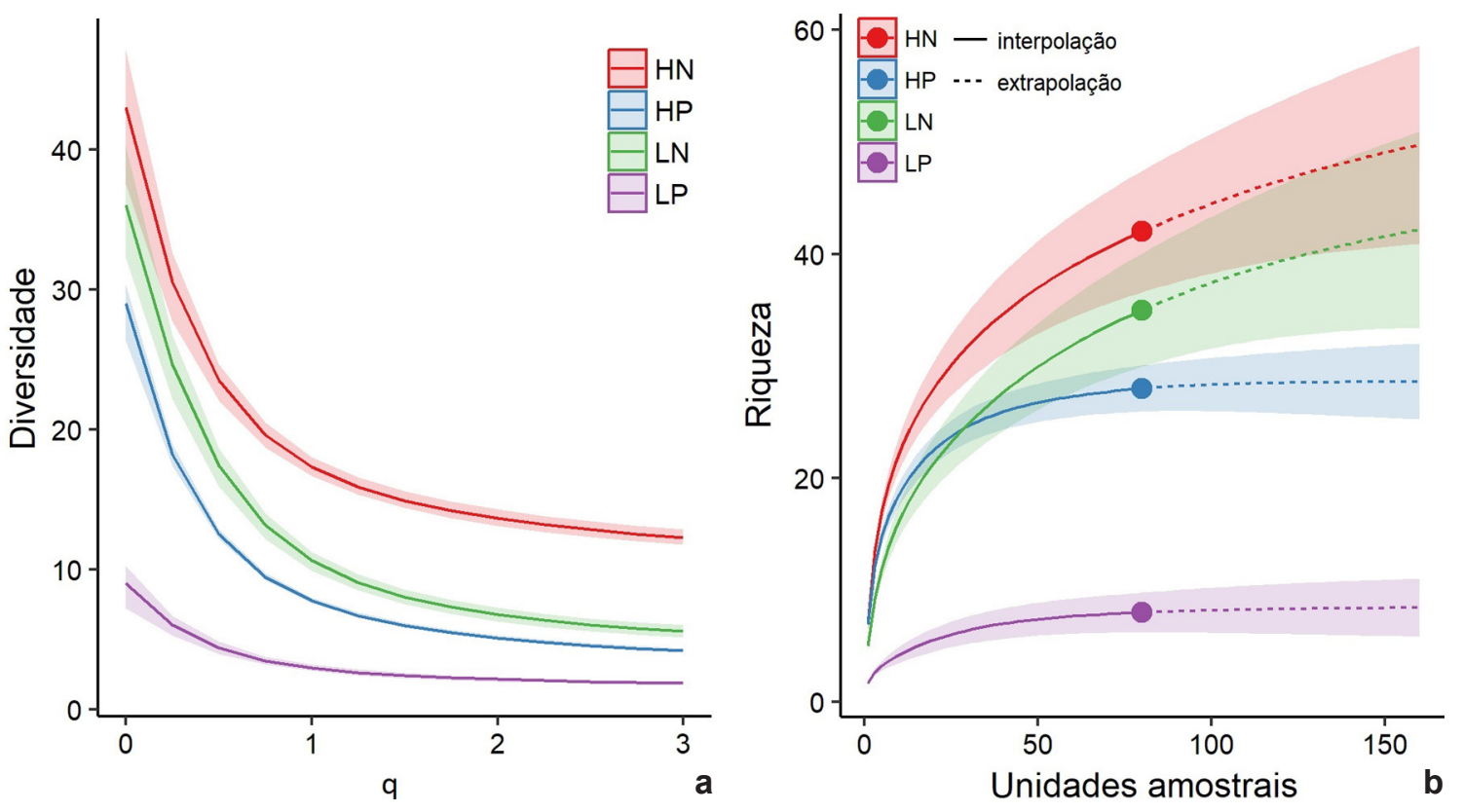

Figura 1 - a. Perfil de diversidade. b. Curvas de interpolação/extrapolação de riqueza de espécies em relação ao número de unidades amostrais. Para os bancos de sementes da Remanescente Florestal Nativo (N) e da área com o Povoamento de Pinus (P), nas diferentes formas de vida avaliada. HN = herbáceas da floresta nativa; HP= herbáceas do povoamento de pinus; $\mathrm{LN}=$ lenhosas da floresta nativa; $\mathrm{LP}=$ lenhosas do povoamento de pinus. Áreas com transparências representam intervalo de confiança a $95 \%$.

Figure 1 - a. Diversity profile. b. Interpolation/extrapolation curves of species richness in relation to the number of sample units. For the seed banks of the Native Remnant Forest $(\mathrm{N})$ and the area with the Pinus Plantation (P), in the different evaluated life forms. HN $=$ herbaceous of native forest; $\mathrm{HP}=$ herbaceous of pine plantation; $\mathrm{LN}=$ woody of native forest; $\mathrm{LP}=$ woody of pine plantation. Areas with transparencies represent $95 \%$ confidence interval.

Em caso de supressão da monocultura de Pinus taeda e necessidade de restauração da floresta nativa, a intervenção antrópica, através do uso de técnicas de restauração ativa, pode ser recomendável dependendo do nível da alteração no ambiente, a fim de aportar maior riqueza de espécies lenhosas.

\section{Agradecimentos}

RTS agradece ao CNPq a bolsa de mestrado (Processo 132070/2015-4) e SJEV agradece a Coordenação de Aperfeiçoamento de Pessoal de Nível Superior (CAPES) a bolsa doutoral.

\section{Referências}

Abreu RCR (2013) Ecologia e controle da invasão de Pinus elliottii no campo cerrado. Tese de Doutorado. Escola de Engenharia da São Carlos da Universidade de São Paulo. São Carlos. 106p.

Araújo MM, Longhi SJ, Barros PLC \& Brena DA (2004) Caracterização de chuva de sementes, banco de sementes do solo e banco de plântulas em Floresta
Estacional decidual ripária Cachoeira do Sul, RS, Brasil. Scientia Florestalis 66: 128-141.

Avila AL, Araujo MM, Longhi SJ \& Gasparin E (2001) Agrupamentos florísticos na regeneração natural em remanescente de Floresta Ombrófila Mista, RS Brasil. Scientia Forestalis 39: 331-342

Baider C, Tabarelli M \& Mantovani W (1999) O banco de sementes de um trecho de uma floresta Atlântica montana (São Paulo - Brasil). Revista Brasileira de Biologia 59: 319-328.

Baider C, Tabarelli M \& Mantovani W (2001) The soil seed bank during Atlantic Forest regeneration in Southeast Brazil. Revista Brasileira de Biologia 61: 35-44.

Brasil (1994) Resolução Conama n 2, de 18 de março de 1994. Define formações vegetais primárias e estágios sucessionais de vegetação secundária, com finalidade de orientar os procedimentos de licenciamento de exploração da vegetação nativa no estado do Paraná. Diário Oficial da União, Brasília, DF, n. 59, 28 mar. 1994, Seção 1. Pp. 4513-4514.

Caldato SL, Floss PA, Da Croce DM \& Longhi SJ (1996) Estudo da regeneração natural, banco de sementes e chuva de sementes na reserva genética 
florestal de Caçador, SC. Ciência Florestal, Santa Maria 6: 27-38.

Calegari L, Martins SV \& Campos LC (2013) Avaliação do banco de sementes do solo para fins de restauração florestal em Carandaí, MG. Revista Árvore 37: 871-880.

Cézar TM (2011) Organogênese direta de Pinus taeda L. Dissertação de Mestrado, Universidade Federal do Paraná, Curitiba. 104p.

Chami LB, Araujo MM, Longhi SJ, Kielse P \& Lúcio AD (2011) Mecanismos de regeneração natural em diferentes ambientes de remanescente de Floresta Ombrófila Mista, São Francisco de Paula, RS. Ciência Rural 41: 251-259.

Chao A \& Jost L (2015) Estimating diversity and entropy profiles via discovery rates of new species. Methods in Ecology and Evolution 6: 873-882.

Chao A (2016) Anne Chao's website - inext. Disponível em <http://chao.stat.nthu.edu.tw/wordpress/ software_download/inext-online/ $>$. Acesso em agosto $20 \overline{1} 6$.

Citadini-Zanette V, Pereira JL, Jarenkow JA, Klein AS \& Santos R (2011) Estrutura da sinúsia herbácea em Floresta Ombrófila Mista no Parque Nacional de Aparados da Serra, Sul do Brasil. Revista Brasileira de Biociências 9: 56-63.

Costalonga SR, Reis GG, Reis MGF, Silva AF, Borges EEL \& Guimarães FP (2006) Florística do banco de sementes do solo em áreas contíguas de pastagem degradada, plantio de eucalipto e floresta em Paula Cândido, MG. Floresta 36: 239-250.

Dalling JW, Swaine MD \& Garwood NC (1998) Dispersal patterns and seed bank dynamics of pioneer trees in moist tropical forest. Ecology 79 : 564-578.

EMBRAPA (2012) Mapa simplificado de solos do estado do Paraná, Brasil. EMBRAPA, Curitiba. Disponível em <http://www.sbcs-nepar.org.br/publicacoes/ publica $\% \mathrm{C} 3 \% \mathrm{~A} 7 \% \mathrm{C} 3 \% \mathrm{~B} 5 \mathrm{es} / \mathrm{mapa}$-simplificadode-solos-do-estado-do-paran\%C3\%A1-detail> Acesso em junho de 2016.

Espíndola MB, Bechara FC, Bazzo MS \& Reis A (2005) Recuperação ambiental e contaminação biológica: aspectos ecológicos e legais. Biotemas, Florianópolis SC 18: 27-38.

Gasparino D, Malavasi UC, Malavasi MM \& Souza I (2006) Quantificação do banco de sementes sob diferentes usos do solo em área de domínio ciliar. Revista Árvore 30: 1-9.

Garwood NC (1989) Tropical soil seed banks: a review. In: Leck MA, Parker VT \& Simpson RL (eds.) Ecology of soil seed banks. Academic Press, London. Pp. 149-209.

GISP - Programa Global de Espécies Invasoras (2005) América do Sul invadida: a crescente ameaça das espécies exóticas invasoras. GISP, Nairobi. 80p.

Gonçalves AR, Martins RCC, Martins IS \& Felfili JM (2008) Bancos de sementes do sub-bosque de Pinus spp. e Eucalyptus spp. na FLONA de Brasília. Cerne 14: 23-32.

Grime JP (1974) Vegetation classification by reference to strategies. Nature 250: 26-31.

Hothorn T, Bretz F \& Westfall P (2008) Simultaneous inference in general parametric models. Biometrical Journal 50: 346-363.

IAPAR - Instituto Agronômico do Paraná (2016a) Cartas climáticas do Paraná. Disponível em $<$ http://www.iapar.br/modules/conteudo/conteudo. php? conteudo=597> . Acesso em 4 julho 2016.

IAPAR - Instituto Agronômico do Paraná (2016b) Médias históricas. Disponível em <http://www.iapar.br/ arquivos/Image/monitoramento/Medias_Historicas/ Lapa.htm>. Acesso em 17 novembro 2016.

ITCG - Instituto de Terras, Cartografia e Geociências (2009) Formações fitogeográficas - estado do Paraná. Disponível em <http://www.itcg.pr.gov. br/arquivos/File/Produtos_DGEO/Mapas_ITCG/ PDF/Mapa_Fitogeografico_A3.pdf $>$ Acesso em junho de 2016 .

ITCG - Instituto de Terras, Cartografia e Geociências (2015) Imagens aréas de 1980 - estado do Paraná. Disponível em < http://www.geo.pr.gov.br/ms4/itcg/ geo.html $>$. Acesso em setembro 2015.

Jankovski T (1996) Estudo de alguns aspectos da regeneração natural induzida em povoamentos de Pinus taeda L. e Pinus elliottii Elgelm. Tese de Doutorado. Universidade Federal do Paraná, Curitiba. $158 \mathrm{p}$.

Kersten RA \& Galvão F (2013) Suficiência amostral em inventários florísticos e fitossociológicos. In: Felfili JM, Eisenlohr PV, Melo MMRF, Andrade LA \& Meira Neto JAA (eds.) Fitossociologia no Brasil: métodos e estudos de caso. Vol. 1. Editora UFV, Viçosa. Pp. 156-173.

Kersten RA, Borgo M \& Galvão F (2015) Floresta ombrófila mista: aspectos fitogeográficos, ecológicos e métodos de estudo. In: Eisenlohr PV, Felfili JM, Melo MMRF, Andrade LA \& Meira Neto JAA (eds.) Fitossociologia no Brasil: métodos e estudos de caso. Vol. 2. Editora UFV, Viçosa. Pp. 156-182.

Kozera C, Dittrich VAO \& Silva SM (2006) Composição florística da floresta ombrófila mista Montana do Parque Municipal do Barigui, Curitiba, PR. Floresta 36: 45-58.

Lopes KP, Souza VC, Andrade LA, Dornelas GV \& Bruno RLA (2006) Estudo do banco de sementes em povoamentos florestais puros e em uma capoeira de Floresta Ombrófila Aberta, no município de Areia, PB, Brasil. Acta Botânica Brasilica 20: 105-113.

Lorenzi H (2008) Plantas daninhas do Brasil: terrestres, aquáticas, parasitas e tóxicas. Instituto Plantarum, Nova Odessa. Pp. 640.

Martins FR \& Batalha MA (2013) Formas de vida, espectro biológico de Raunkiaer e fisionomia da vegetação. In: Felfili JM, Eisenlohr PV, Melo 
MMRF, Andrade LA \& Meira Neto JAA (eds.) Fitossociologia no Brasil: métodos e estudos de caso. Vol. 1. Editora UFV, Viçosa. Pp. 44-85.

MINEROPAR - Minerais do Paraná (2001) Atlas geológico do estado do Paraná. Mineropar, Curitiba. Pp. 38.

Narvaes IS, Brena DA \& Longi SJ (2005) Estrutura da regeneração natural em Floresta Ombrófila Mista na Floresta Nacional de São Francisco de Paula, RS. Ciência Florestal 15: 331-342.

Ne'eman G \& Izhaki I (1999) The effect of stand age and microhabitat on soil seed banks in Mediterranean Aleppo pine forests after fire. Plant Ecology 144: 115-125.

Nóbrega AMF, Valeri SV, Paula RC, Pavani MCMD \& Silva SA (2009) Banco de sementes de remanescentes naturais e de áreas reflorestadas em uma várzea do rio Mogi-Guaçu, SP. Revista Arvore 33: 403-411.

Oliveira Junior RS \& Constantin J (2001) Plantas daninhas e seu manejo. Ed. Agropecuária, Guaíba. $362 p$.

R Core Team (2016) A language and environment for statistical computing. R Foundation for Statistical Computing, Vienna. Disponível em $<$ http://www.Rproject.org/>. Acesso em agosto de 2016.

Richardson DM \& Bond WJ (1991) Determinants of plant distribution: evidence from pine invasions. The American Naturalist 137: 639-668.

Roderjan CV, Galvão F, Kuniyoshi YS \& Hatschbach GG (2002) As unidades fitogeográficas do estado do paraná, Brasil. Ciência \& Ambiente 24: 75-92.

Schorn LA, Fenilli TAB, Kruger A, Pellens GC, Budag JJ \& Nadolny MC (2013) Composição do banco de sementes no solo em áreas de preservaçáo permanente sob diferentes tipos de cobertura. Floresta 43: 49-58.

Seubert RC, Maçaneiro JP, Budag JJ, Fenilli TAB \& Schorn LA (2016) Banco de sementes do solo sob plantios de Eucalyptus grandis no município de Brusque, Santa Catarina. Floresta 46: 165-172.
Silva-Weber AJC, Nogueira AC, Carpanezzi AA, Galvão F \& Weber SH (2012) Composição florística e distribuição sazonal do banco de sementes em Floresta Ombrófila Mista Aluvial, Araucária, PR. Pesquisa Florestal Brasileira 32: 77-91.

Siqueira LP (2002) Monitoramento de áreas restauradas no interior do estado de São Paulo, Brasil. Dissertação de Mestrado, Escola Superior de Agricultura Luiz de Queiroz, Piracicaba. 116p.

Souza VC \& Lorenzi H (2008) Botânica sistemâtica: guia ilustrado para identificação das famílias botânicas da Fanerógamas nativas e exóticas do Brasil. Instituto Plantarum, Nova Odessa. 703p.

Souza ML, Nogueira AC, Macedo RLG, Sanquetta CR \& Venturi N (2011) Estudos de um banco de sementes no solo de um fragmento florestal com Araucaria angustifolia no estado do Paraná. Floresta 41: 335-346.

Tabarelli M \& Mantovani W (1999) Regeneration of a neotropical montane forest following slash-andburn (São Paulo, Brazil). Revista Brasileira de Biologia 59: 239-250.

Vargas L \& Bernardi J (2003) Manejo de plantas daninhas na produção orgânica de frutas (Circular Técnica, n. 45). Embrapa Uva e Vinho, Bento Gonçalves. 11p.

Venables WN \& Ripley BD (2002) Modern applied statistics with S. $4^{\text {th }}$ ed. Springer, New York. 495p

Vinha D (2008) Banco de sementes em áreas com diferentes graus de perturbação no Parque Estadual das Fontes do Ipiranga, em São Paulo, SP. Dissertação de Mestrado. Instituto de Botânica de São Paulo, São Paulo. 105p.

Zanchetta D \& Diniz FV (2006) Estudo da contaminação biológica por Pinus spp. em três diferentes áreas na estação ecológica de Itirapina. Revista do Instituto Florestal 18: 1-14.

Ziller SR \& Galvão FA (2002) Degradação da estepegramíneo lenhosa no Paraná por contaminação biológica de Pinus elliottii e P. taeda. Floresta 32: 41-47. 